\title{
Understanding the neurobiological basis of behavior: a good way to go
}

\author{
Carmen Sandi* \\ Brain Mind Institute, Ecole Polytechnique Federale de Lausanne (EPFL), Lausanne, Switzerland \\ *Correspondence: carmen.sandi@epfl.ch
}

Not so long ago, Behavioral Neuroscience was considered a 'soft' science, with the search for new molecules and the cellular functions of the molecules being the leading model illustrating what was considered essential to be studied to understand brain function. This has drastically changed in the last decade, particularly with the publication of the human genome and later on that of other species (notably the mouse one). The time came when the need for placing molecules in 'context' and identifying the role of the molecules in the behaving animal became clearly apparent.

Such a development in the Neuroscience panorama has placed Behavioral Neuroscience at quite a central place. It is clear now that we cannot determine whether a particular molecule is involved in memory formation just by testing the molecule's effects through in vitro approaches (by evaluating, for example, whether it influences neurite outgrowth in neuronal cultures, or long-term potentiation (LTP) in brain slices, just to mention two of the approaches most frequently used in this type of screening). Although the earlier studies showed that effects on learning should match the effects found for LTP, nowadays there are many examples in the literature of dissociations between the effects exerted on LTP and on memory tasks by specific molecules, most frequently with one (LTP or learning) being affected and not the other (Shimshek et al., 2006), or even each of them displaying opposite effects (Rutten et al., 2008). In fact, the lack of prediction from one system to the other makes total sense. Reproduction of the dynamic complexity that characterizes every single organism in a piece of brain tissue now appears to be a naïve oversimplification. Many are the missing elements (hormones, cytokines, to only cite a few, well established ones), that play a role in behavior and cognition in the entire animal when only a piece of tissue is examined.

However, proposing a central role for Behavioral Neuroscience should not disregard the fact that Human Neuroscience is taking strong positions in the domains that have been classically defined within the scope of Behavioral Neuroscience. The non-inclusion of human studies in the field of Behavioral Neuroscience is, in my view, an artificial segregation. As stated in the mission statement of Frontiers in Behavioral Neuroscience, I consider that this field includes the study of the neurobiological basis of behavior both in animals and humans. Now, we can be informed of the neurobiological basis of behavior

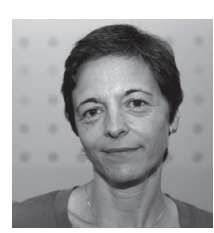

Carmen Sandi received her PhD in 1988 in Behavioral Neurobiology. After postdoctoral work at the Cajal Institute in Madrid, INSERM-University of Bordeaux, and The Open University, UK, she was recruited as Professor of Psychobiology by UNED, Madrid, in 1996. Since 2003, she is Professor at the Brain Mind Institute, EPFL, Switzerland, where she heads the Laboratory of Behavioral Genetics. She is interested in understanding how stress affects brain function, behavior and cognition. in humans through a comprehensive number of neurobiological approaches (including lesions, brain stimulation, neuroimaging, human brain banks, and genetic analyses - from single-nucleotide polymorphisms to genome - and epigenome - wide analyses) complementing progressively refined behavioral analyses [with virtual reality approaches already being an actual possibility to increase the ecological value of behavioral testing (Slater et al., 2006)]. In fact, the question 'why do we need mice if nowadays we can virtually do everything in humans?' is becoming not so infrequent. Even though answering to the question is not an easy task, studies on animals have still much to offer to the understanding of the neurobiological basis of behavior. In no way, human researchers can dream to manipulate variables and conditions and to get to the fine detailed cellular, molecular, and functional approaches in the studies that animal research allows. In fact, the good shape of the two subfields (animal and human Behavioral Neuroscience) is clearly materializing in mutual benefit (Carola et al., 2008; de Quervain et al., 2007) and the reciprocal interaction comes as a momentum for the field as a whole.

Let's now focus on what still seems to be the core study in the field of Behavioral Neuroscience: animal and, to a large extent, rodent studies. What are the challenges we are facing now? Being aware that any attempt to answer such a question can only be subjective, I will briefly comment on the issues that I am particularly sensitive to, without any intention to be comprehensive.

The first issue is certainly the urgent need for a refining of behavioral testing, and includes many issues from continuing improvement of the accuracy of measures to progressively automatizing the whole testing process. One idea would be to develop more complex housing environments including a range of testing opportunities under social conditions, with the main goals being to avoid taking out animals from their normal environment, minimizing differences due to human manipulation, and improvement in standardization. Although there is already an interesting development in the market, known as the Intellicage (Knapska et al., 2006), there is still huge room for expansion. Refinement of the behavioral testing would also include the development of new validated tests to enhance and improve the measurement of behavior. There is also an imminent need for improving and expanding the animal models of psychopathology. The development of models with good face validity can greatly benefit from automated approaches (taking out the animal from its home cage for a restricted time per day to be submitted, for example, to stress-induction procedures with the aim of eventually modeling depression is clearly less 'ecologically' valid than organizing the delivery of stressors in their 'natural' environment according to a controlled scheme). Considering the sophistication of analyses reached at other domains of Neuroscience (such as the high resolution of current 
genomic analyses, electron or two-photon microscopes, to give just a few examples), enriching the behavioral repertoire during testing and the precision of measurement is an imperative soon to be vigorously tackled by the field of Behavioral Neuroscience. The depth of insight that one can achieve at the neurobiological levels is directly dependent on the quality of the behavioral analyses that can be implemented.

Another important issue is to solve the existing difficulties in reproducibility of behavioral results. Important efforts are being made to identify the operating procedures and variables that either allow or prevent reproducibility of results across both time and laboratories (Mandillo et al., 2008; Wolfer et al., 2004), but there is still much to be done in this regard.

The issue of individual differences frequently regarded as an inconvenient problem is, in my view, a great opportunity for this field to develop. The fact that we - humans and animals - are so different in our behavioral reactions, abilities, and motivations, is such a big reality that pretending to have uniform animal models to understand 'the' neurobiological basis of behavior does not seem to be the definitive approach. Understanding and defining what underlies such behavioral complexity are by far a richer goal than denying it with 'statistics of the mean'. In this regard, the standard approach to develop genetically engineered animals (knockouts, transgenic) in a single inbred mouse strain informs about the role of the gene under study for the 'specific genome' of a 'particular background mouse strain', without allowing any firm conclusion about the role for such a gene in other genomic 'contexts' (i.e., genetically different individuals). The limitation can now be overcome by including genetic variations in our experimental design with the application of recently developed resources, such as chromosome substitution strains and the Collaborative Cross project, or the application of whole-genome analysis in outbred strains (Flint et al., 2005). Alternatively, profiling the animals according to personality-like traits is another highly promising tool to account for variations in animal behavior and corresponding neurobiological correlates (Belin et al., 2008; Sandi et al., 2008).

\section{REFERENCES}

Belin, D., Mar, A. C., Dalley, J. W., Robbins, T.W., and Everitt, B.J. (2008). High impulsivity predicts the switch to compulsive cocaine-taking. Science 320, 1352-1355.

Carola, V., Frazzetto, G., Pascucci, T., Audero, E., Puglisi-Allegra, S., Cabib, S., Lesch, K. P., and Gross, C. (2008). Identifying molecular substrates in a mouse model of the serotonin transporter $\times$ environment risk factor for anxiety and depression. Biol. Psychiatry 63, 840-846.

deQuervain,D.J.,Aerni,A., and Roozendaal, B. (2007). Preventive effect of betaadrenoceptor blockade on glucocorticoid-induced memory retrieval deficits. Am. J. Psychiatry 164, 967-969.

Flint, J., Valdar, W., Shifman S., and Mott, R. (2005).Strategies for mapping and cloning quantitative trait genes in rodents. Nat. Rev. Genet. 6, 271-286.
Knapska, E., Walasek, G., Nikolaev, E., Neuhäusser-Wespy, F., Lipp, H. P., Kaczmarek, L., and Werka, T. (2006). Differential involvement of the central amygdala in appetitive versus aversive learning. Learn. Mem. 13, 192-200.

Mandillo,S., Tucci,V.,Hölter,S.M.,Meziane, H., Banchaabouchi, M. A., Kallnik, M., Lad, H. V., Nolan, P. M., Ouagazzal, A. M., Coghill, E. L., Gale, K., Golini, E., Jacquot, S., Krezel, W., Parker, A., Riet, F.,Schneider, I., Marazziti, D., Auwerx, J., Brown,S.D., Chambon, P., Rosenthal, N., Tocchini-Valentini, G., and Wurst, W. (2008). Reliability, robustness, and reproducibility in mouse behavioral phenotyping: a cross-laboratory study. Physiol. Genomics 34, 243-255.

Rutten, K., Misner, D. L, Works, M., Blokland, A., Novak, T. J., Santarelli, L., and Wallace, T. L. (2008). Enhanced long-term potentiation and impaired learning in phosphodiesterase

In continuance with the issues related to complexity, one of the great challenges will be to understand how gene and environment interact to produce behavior. The field of epigenetic regulation of behavior provides some striking solutions (Tsankova et al., 2007) but the whole picture is far from being understood.

In fact, the ultimate challenge is probably to understand the complexity inherent to each of the neurobiological levels, particularly the interrelation of the levels in the emerging property of generating behavior. Can we ever understand the integrated sequence of events (cellular, molecular, and network function) that lead to a particular behavioral manifestation? To illustrate the difficulty, just think of the rising myriad of molecules reported to be 'required' for memory formation. How can we explain that so many molecules are essential when we frequently learn that removing or temporally silencing a particular molecule can turn animals 'amnesic'? And if this is true when just remaining at a specific level (molecular), how does it translate on the corresponding changes that should also occur at each of the other biological levels that regulate behavior? Understanding this ambitious goal requires meticulous knowledge not only of molecular pathways, but also of their cellular, brain, and individual specificity (but be reminded that we took 'molecules' as an example; the same exhaustive characterization will be needed also for elements from other levels of analyses).

The understanding of such complexity is impossible to achieve without the development of sophisticated non-invasive approaches allowing the finest temporal and spatial resolution for each of the participating elements (cellular, molecular, and network function), thus overcoming current limitations on the dissection of so many systems for the study of the neurobiological basis of behavior that still can only be targeted through ex vivo approaches. Eventually, assembling the puzzle will require bringing together many pieces of knowledge generated by a large diversity of Neuroscience subdisciplines (from molecular, cellular, and circuit to systems Neuroscience, among others), finding their place and function in behaving individuals, and understanding their combinatorial properties with the twist of bioinformatics and modeling approaches.

4D-knockout (PDE4D) mice. Eur. J. Neurosci. 28, 625-632.

Sandi, C., Cordero, M. I., Ugolini, A. Varea, E., Caberlotto, L., and Large, C. (2008). Chronic stress-induced alterations in amygdala responsiveness and behavior: modulation by trait anxiety and CRF systems. Eur. J. Neurosci. 28 1836-1848.

Shimshek, D. R., Jensen, V., Celikel, T., Geng, Y., Schupp, B., Bus, T., Mack, V., Marx, V., Hvalby, Ø., Seeburg, P. H., and Sprengel, R. (2006). Forebrain-specific glutamate receptor $B$ deletion impairs spatial memory but not hippocampal field long-term potentiation. J. Neurosci. 26, 8428-8440.

Slater,M.,Antley,A., Davison,A.,Swapp, D., Guger, C., Barker, C., Pistrang, N., and Sanchez-Vives, M. V. (2006). A virtual reprise of the Stanley Milgram obedience experiments. PLoS ONE 1, e39.
Tsankova, N., Renthal, W., Kumar, A., and Nestler, E. J. (2007). Epigenetic regulation in psychiatric disorders Nat. Rev. Neurosci. 8, 355-367.

Wolfer, D. P., Litvin, O., Morf, S., Nitsch, R. M., Lipp, H. P., and Würbel, H. (2004). Laboratory animal welfare: cage enrichment and mouse behaviour. Nature 432 821-822.

Received: 12 October 2008; published online: 15 December 2008 Citation: Front. Neurosci. (2008) 2, 2: 129 130. doi: 10.3389/neuro.01.046.2008 Copyright: (c) 2008 Sandi. This is an openaccess publication subject to an exclusive license agreement between the authors and the Frontiers Research Foundation, which permits unrestricted use, distribution, and reproduction in any medium, provided the original authors and source are credited. 